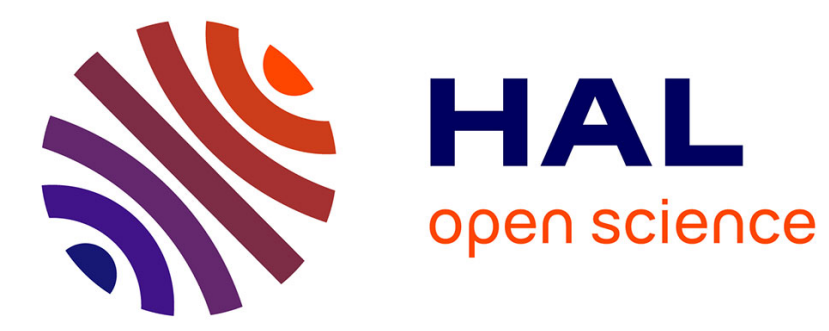

\title{
Les usages de Twitter au festival de Cannes. Pratiques numériques et construction des opinions esthétiques
}

\author{
Myriam Dougados, Jean-Louis Fabiani, Julien Gaillard, Yonathan Portilla
}

\section{To cite this version:}

Myriam Dougados, Jean-Louis Fabiani, Julien Gaillard, Yonathan Portilla. Les usages de Twitter au festival de Cannes. Pratiques numériques et construction des opinions esthétiques. Culture et Musées, 2021, pp.89-106. 10.4000/culturemusees.639 . hal-03265452

\section{HAL Id: hal-03265452 \\ https://hal.science/hal-03265452}

Submitted on 20 Jun 2021

HAL is a multi-disciplinary open access archive for the deposit and dissemination of scientific research documents, whether they are published or not. The documents may come from teaching and research institutions in France or abroad, or from public or private research centers.
L'archive ouverte pluridisciplinaire $\mathbf{H A L}$, est destinée au dépôt et à la diffusion de documents scientifiques de niveau recherche, publiés ou non, émanant des établissements d'enseignement et de recherche français ou étrangers, des laboratoires publics ou privés. 


\section{OpenEdition}

Journals

\section{Culture \& Musées}

Muséologie et recherches sur la culture

$24 \mid 2014$

Démocratisation culturelle et numérique

\section{Les usages de Twitter au festival de Cannes. Pratiques numériques et construction des opinions esthétiques}

Twitter Usage during the Cannes Cinema Festival. Digital Usages and Construction of Aesthetic Opinions Los usos de Twitter en el festival de Cannes. Las prácticas digitales y la construcción de opiniones estéticas

Myriam Dougados, Jean-Louis Fabiani, Julien Gaillard et Yonathan PorTILLA

p. $89-106$

https://doi.org/10.4000/culturemusees.639

\section{Résumés}

Français English Español

Cet article est une contribution à l'analyse des effets des réseaux sociaux sur la formation des goûts et des opinions en matière culturelle. En prenant l'exemple des usages de Twitter au Festival de Cannes par trente-deux professionnels partenaires de Vodkaster qui jouent ensemble sur la scène cannoise et en s'appuyant principalement sur une analyse lexicale, on peut mettre au jour trois notions structurantes : celle d'outsider numérique - la déviance étant constitutive de l'événement -, celle de leadership fondée sur la fidélité du lectorat, et celle d'un quatrième cercle de spectateurs qui construit son expérience du Festival par procuration. Les réseaux sociaux numériques constituent ainsi à la fois une ressource aisément exploitable pour l'analyse des publics et une forme spécifique de la sociabilité festivalière.

This article is a contribution to the analysis of the effects of social networks on the shaping of taste and opinions concerning cultural matters. Taking as an example the Twitter usage of thirty-two professional partners of Vodkaster who play with each other on the Cannes stage and using mainly lexical analysis, one can shed light on three structural concepts: the digital outsider - his deviance being a central feature of the event, leadership - based on faithful readership, and the fourth circle 
of spectators who experience the festival by proxy. Thus, digital social networks are both an easily mobilized resource for the analysis of audience and a specific form of festival-goer's sociability.

Este artículo es una contribución al análisis de los efectos de las redes sociales sobre la formación de los gustos y de las opiniones en materia cultural. Tomando el ejemplo de los usos de Twitter en el festival de Cannes de treinta y dos profesionales (socios de Vodkaster) que tocan juntos en la escena local, y apoyados fundamentalmente en un análisis lexical, podemos dar a luz tres nociones estructurantes : la de outsider numérico (la desviación constitutiva del acontecimiento), la de liderazgo fundada sobre la fidelidad de los lectores y la del cuarto círculo de espectadores que construye su experiencia del festival en su nombre. Las redes sociales constituyen a la vez un recurso fácilmente explotable para el análisis de los públicos y una forma específica de la sociabilidad del festival.

\section{Entrées d'index}

Mots-clés : Twitter, réseaux sociaux, public, lexicologie, goût, festival

Keywords: Twitter, social networks, audience, lexicology, taste, festival

Palabras clave: Twitter, redes sociales, públicos, lexicología, gusto, festival

\section{Notes de la rédaction}

Manuscrit reçu le : 7 février 2014

Version révisée, reçue le : 5 août 2014

2e version révisée, reçue le : 21 août 2014

Article accepté pour publication le : 3 septembre 2014

\section{Texte intégral}

Que viennent introduire ou'modifier les réseaux sociaux dans le jeu de l'influence en matière d'opinion ou de goût, particulièrement dans le domaine culturel ? Comment se transmet et se diffuse un point de vue sur des œuvres, des styles ou des situations?

On peut être conduit à majorer le pouvoir des nouvelles formes de communication, dans la tradition d'un optimisme technologique qui voit toujours dans l'innovation technique la source privilégiée des transformations sociales. On peut au contraire être tenté de rabattre toutes les nouveautés apparentes vers les catégories traditionnelles de l'analyse de l'influence, dans le prolongement des analyses classiques d'Elihu Katz et Paul Lazarsfeld (Katz \& Lazarsfeld, 1954). Les remarques qui suivent ne prétendent pas contribuer à la construction d'une théorie de l'influence dans le domaine culturel. Elles se situent plutôt à la croisée de deux lignes de recherche : la première prolonge des travaux collectifs menés dans la durée sur la forme festival et sur la spécificité des interactions qu'elle suscite en matière d'évaluation des œuvres et des événements. La seconde est celle de la prise en compte des usages des réseaux sociaux dans les nouvelles stratégies de présentation de soi et de mobilisation de l'opinion. Les réseaux tendent en effet à modifier la structure de l'espace public, particulièrement en ce qu'ils permettent l'instantanéité, la multiplicité et la diversité (au moins apparente) des points de vue.

3 Parmi les réseaux sociaux ayant vu le jour ces dernières années, Twitter est sans doute un de ceux qui ont connu le développement le plus spectaculaire en proposant à ses usagers de publier des messages en 140 signes pouvant renvoyer à d'autres ressources numériques, d'autres réseaux, et de multiplier quasi indéfiniment les possibilités d'atteindre des individus et de les faire entrer dans le cercle des commentaires. Twitter est caractérisé par la coexistence d'émetteurs et de récepteurs, ces derniers étant définis de manière intéressante comme des « suiveurs » (followers). On peut suivre un émetteur en silence, réagir à ses propos et les faire partager. Le nombre de followers, comme le nombre d'amis sur d'autres types de réseaux sociaux, est une forme de ressource ou de capital qui constitue en tant que tel, ou sous une forme cumulée avec d'autres réseaux, un indicateur réputationnel. Notoriété et popularité peuvent donc être évaluées, quantitativement si l'on peut dire, à partir du nombre de followers, mais aussi à partir du 
nombre des commentaires et des partages que les tweets suscitent. La dimension réputationnelle concerne aussi bien les citoyens ordinaires - parmi lesquels les adolescents constituent une catégorie importante - que les faiseurs d'opinion, qui peuvent par leurs tweets court-circuiter des formes plus établies de communication, y compris électroniques, et se prévaloir d'une dynamique de la spontanéité, de l'instantanéité et de l'événementialité pour tenter d'accroître ce qu'on peut identifier comme une nouvelle forme de capital social. D’une manière générale, l'objet de ces réseaux sociaux est en effet de constituer des cercles d'influence reposant sur des intérêts communs et d'attirer à soi, en une phrase - qui, au regard de sa brièveté, pourrait être qualifiée d' « accroche » ou de « slogan »-, l'espace d'attention (Collins, 2000).

4 Le tweet est donc parfaitement adapté au traitement de l'actualité la plus chaude : il permet de rendre compte de l'hyper-présent dans sa dimension la plus concentrée. Il est fondé non sur le raisonnement ou les techniques de persuasion, mais sur la formule choc, destinée à faire mouche sans délai. L'aspect temporel est ici essentiel, de même que l'effet de réel que permet l'attestation de la présence - je vous dis par mon tweet que je suis bien à Cannes. Le tweet est un concentré de temps, la mise en forme d'une intensité. Le festival densifie la temporalité en imposant une scansion particulière et une saturation des plages disponibles par accumulation des événements aussi bien que des possibilités d'événement. La forme festival reproduit dans un temps et un espace donnés une organisation sociale, et configure un espace public spécifique, au sens où elle met en jeu l'engagement de chacun dans le dispositif festivalier.

5 Elle donne lieu ainsi à un concentré de vie citoyenne, d'usages et d'interactions. Par ailleurs, les festivals, qui investissent des lieux préalablement habités, suscitent des cohabitations où les intérêts divergent et provoquent des frottements qui font émerger des façons d'occuper le territoire festivalier. Dans un festival comme celui de Cannes, où l'événement lui-même devient un spectacle dont on peut être le public, les réseaux sociaux, plus qu'ailleurs, sont un moyen d'identification de l'événement, et même de l'événementialité de l'événement. Il peut se passer plus ou moins de choses au cours d'un événement donné : une bretelle de soutien-gorge se rompt pendant la montée des marches, un acteur commet un impair, etc. Toute intervention sur les réseaux sociaux est une manière de se faire reconnaître et de se faire aimer, comme l'est toute tentative de communication verbale ou non verbale. Le festivalier est un témoin dont l'influence potentielle tient à sa situation au cour de l'événement.

Extraits des relevés de messages postés sur Twitter via le site Vodkaster :

Pour le moment vous ne manquez rien a \#cannes2012 et s'il se passe des trucs en ce moment je ne suis pas au courant Je n'ai toujours rien à dire ne m oubliez pas

En prenant pour terrain le Festival de Cannes, nous avons fait le choix d'exploiter la culture des écrans propre au public de ce Festival, un public qu'on peut supposer plus sensible aux nouvelles technologies. Le Festival de Cannes étant lui-même un lieu historique de production de liens sociaux et de développement de réseaux, il nous a semblé que le public festivalier était un analyseur privilégié du jeu des interactions électroniques.

7 La forme festival démultiplie les capacités spectatorielles dans le sens où chacun, présent dans l'espace et le temps festivalier, devient public de l'événement et participe à la constitution d'un cercle de spectateurs, plus ou moins perceptible comme tel, par ses protagonistes aussi bien que par ceux qui les observent. Les regardeurs sont regardés et le savent : une telle mise en abîme exige de chacun une compréhension de son statut de festivalier et une approche réflexive des usages attachés à la position de spectateur. Le Festival de Cannes multiplie les injonctions à l'égard de ses publics, de l'étiquette vestimentaire à l'affichage des jugements de goût. Il édicte à chacun la conduite à tenir et produit des formes d'identification contraignantes aussi bien que des hiérarchies symboliques. En cela, le Festival de Cannes est un lieu qui met en question spécifiquement 
la porosité des différents cercles de spectateurs, notamment par son système d'accréditation.

8 En effet, l'accès au Festival est réglementé par un système d'accréditation qui ouvre et ferme les portes festivalières selon le statut professionnel. Chacun appartient ainsi à un cercle de spectateurs construit à partir de la reconnaissance que lui délivre l'instance festivalière. Alors que l'ensemble du dispositif festivalier se veut très didactique et développe de nombreux supports qui guident et accompagnent le spectateur dans ses circulations, la codification des accréditations est peu lisible et ne permet que partiellement de se repérer dans l'organisation de l'espace public ainsi produit. Ce point obscur signale la façon dont le rapport au Festival s'envisage dans une logique de proximité au monde du cinéma et provoque ainsi des comportements spécifiques liés au fait que le festivalier ne sait jamais vraiment qui est son voisin de fauteuil. L'accès y devient un enjeu de reconnaissance dans le monde du cinéma qu'il convient de maitriser afin de ne pas perdre la face (Goffman, 1967) devant une porte qui se ferme. La logique de réseau inhérente à un forum professionnel devient alors un jeu de piste dans l'apprentissage des codes sociaux propres au festival. Le festival est un lieu aussi chaleureux que dangereux, une récompense aussi bien qu'une épreuve.

$9 \quad$ Les réseaux sociaux apparaissent au contraire comme un moyen de créer des liens qui ne sont pas contraints en fonction des limites inhérentes aux cercles définis par l'institution ou par les formes de capital social ou culturel possédées. Nous avons donc voulu observer si ces nouveaux outils permettaient d'identifier de nouvelles grilles relationnelles construites autour d'un système d'affinités électives plutôt que produites par les déterminations sociales et, si possible, de mesurer l'écart entre les relations virtuelles et la vie quotidienne d'un festivalier.

10 Sur ces réseaux cohabitent des communautés d'affinités et des communautés d'intérêts dans une porosité au moins apparente, qui est pour une bonne part constituée par la logique de l'événement. En cela, les réseaux sociaux intensifient une des propriétés de la forme festival dans sa dimension de confrontation des opinions. Il nous a donc semblé pertinent d'étudier comment la forme festival est matérialisée sur les réseaux sociaux et comment elle reproduit, infléchit ou contredit l'organisation sociale du festival.

11 Il s'agit pour ce faire de s'appuyer sur ces réseaux, qui conservent une mémoire de l'événement, pour développer une analyse des terrains festivaliers qui, par leur concentration dans le temps et l'espace, rendent l'observation des situations difficile parce que les interactions sont fugaces. En effet, les réseaux sociaux gardent les traces de l'événement et permettent de poursuivre l'enquête en dehors de l'instantanéité du terrain.

12 La porosité des cercles cannois est relative au manque de lisibilité des situations sociales d'interaction. L'absence de repères quant au statut de son voisin de fauteuil ou de celui avec qui on va partager quelques heures de file d'attente demande, à qui attend du Festival une ouverture sur le monde du cinéma, une sociabilité de tous les instants aussi bien qu'une forme de "sens pratique ». Au-delà de la popularité des stars, il existe des popularités moins brillantes mais décisives en ce qu'elles permettent de faire son chemin dans l'univers festivalier qui va bien au-delà du simple visionnage de films. Chacun vient à Cannes avec ses propres objectifs. Pour certains l'enjeu sera de croiser une star ; même de seconde zone, dans une déambulation sur La Croisette ; pour d'autres, ce sera de participer, aux côtés des grands, aux cérémonies médiatiques du Festival ; pour d'autres enfin, de provoquer les rencontres et de se faire reconnaître par ce milieu professionnel. Pour chacun, il s'agit de marquer sa présence cannoise et de la rendre publique ${ }^{1}$.

13 Sur Twitter, chaque fil peut être déroulé pour ce qu'il est, une trace instantanée et unique. Mais ce fil, relié aux autres, délimite le territoire d'une communauté cannoise émergente et en dévoile les hiérarchies. On peut alors se demander si les propos sont les mêmes selon qu'ils sont postés du haut des marches ou au pied du palais, si l'on exprime de la même manière le passage de Robert De Niro sur La Croisette selon qu'on est accrédité ou badaud et si, finalement, nos messages ne dévoilent pas plus que ce qu'ils disent sur notre présence festivalière. La Croisette, le palais, les marches... : des lieux ont 
construit l'imaginaire du Festival de Cannes et en produisent une géographie symbolique. Pourtant, le territoire festivalier s'étend bien au-delà de ce périmètre médiatique et l'on voit, sur le terrain, se dessiner une cartographie du Festival dont les frontières sont celles, mouvantes, des circulations de ses publics. En cela, les réseaux sociaux participent de cette mise en lumière de leur présence festivalière et deviennent un moyen de mesurer la porosité relative des cercles festivaliers. En effet, avant l'apparition des réseaux sociaux, certains échanges restaient, sinon dans le domaine de l’intime, au moins dans le cercle privé. Ils s'affichent aujourd'hui dans le domaine public et sont exploités comme un vecteur de connaissance, de reconnaissance et de publicisation de $\mathrm{soi}^{2}$. Ces outils, qui laissent une trace et construisent ensemble une mémoire d'un terrain festivalier par définition fugitif, permettent une observation du terrain en dehors du temps cannois. L'étude des traces, des échos, aussi bien que la mise en perspective de ces instantanés, doivent pouvoir nous amener à construire une lecture plus adéquate des objets cannois et d'en définir un maillage, des circulations et des territoires. Nous disposons pour cela d'une base de données contenant l'ensemble des tweets postés durant les éditions 2012 et 2013 sur le site Vodkaster.

\section{Insiders, outsiders?}

Le site Vodkaster a recréé le microcosme cannois dans un dispositif intitulé "Cannes Inside » qui rassemble trente-deux festivaliers, les insiders, dont les tweets sont relayés sur le site. L'ensemble de ces données est accessible via l'API ${ }^{3}$ de

Vodkaster, avec qui nous travaillons en collaboration dans le cadre de la structure fédérative de recherche Agorantic de l'université d'Avignon autour de l'analyse de la microcritique.

Développé depuis 2011, ce dispositif semble vouloir contribuer à la construction électronique des cercles cannois par le rassemblement d'une communauté festivalière organisée selon une diversité de statuts sociaux. Ces insiders sont un groupe d'une trentaine de festivaliers aux âges, statuts sociaux et postures divers, chargés de relayer leurs expériences en temps réel, via les réseaux sociaux. L'appellation d' insiders », que l'on peut traduire par " initiés », implique que l'on touche au cœur de l'expertise festivalière et autorise l'idée, pour les followers, qu'ils vont pouvoir faire une intrusion dans l'intimité festivalière et accéder à la possibilité de vivre le Festival par procuration. Le visuel du dispositif « Cannes Inside », dont le « i » est figuré par un trou de serrure, renforce ce sentiment de voir l 'invisible. Il s'agit là d'une forme d'effraction symbolique rendue possible par les réseaux sociaux.

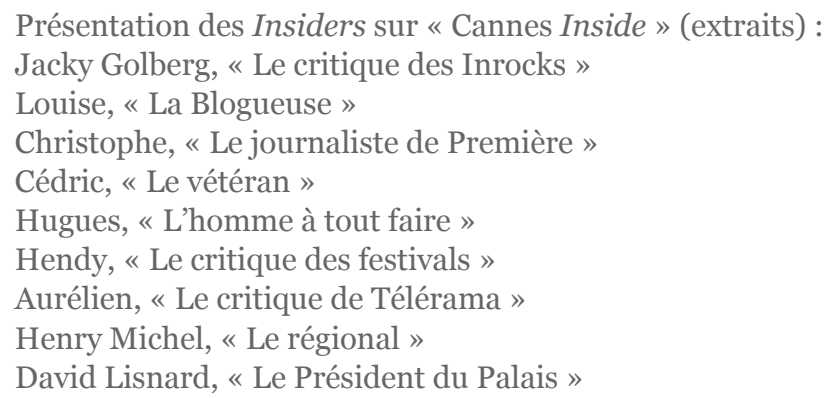

Ce sont donc trente-deux insiders qui couvrent la période festivalière, neuf femmes et vingt-trois hommes. Pour la majorité d'entre eux, il s'agit de professionnels des partenaires de Vodkaster ou de blogueurs. Vodkaster affirme d'ailleurs avoir « casté » ses insiders. Certains participent à l'événement pour la première fois, d'autres en sont déjà à leur trente-cinquième édition, mais ils ont en moyenne neuf années de fréquentation festivalière. Chaque insider est référencé par son prénom - de façon plus exceptionnelle s'y ajoute le nom de famille - un statut généralement professionnel (journaliste, 
producteur...) et le nombre d'années de Festival. Chaque statut est précédé par « le » ou « la » et souvent complété par un qualificatif : « La journaliste timide. »Cette présentation de soi induit le caractère unique de chaque insider mais atteste aussi d'une volonté de représentation plus globale d'un type de festivalier, voire de capacité d'identification au personnage. Car c'est bien cela qui nous est proposé : il s'agit de suivre les pérégrinations festivalières d'une trentaine de personnages qui jouent, ensemble - les échanges de tweets entre eux sont fréquents -, sur la scène du Festival de Cannes.

La majorité de ces insiders sont qualifiés de journalistes, qu'ils soient indiqués comme tels ou comme critiques. La deuxième catégorie professionnelle représentée est celle du milieu du cinéma - de l'actrice au distributeur. Enfin, quelques profils plus isolés sont présentés, souvent liés à un statut autre que professionnel (le régional, le vétéran, le cinéphile...). Nous sommes donc confrontés à une logique de types.

Au regard des chiffres donnés par le Festival quant à la répartition des accréditations selon les catégories professionnelles, $26 \%$ des insiders sont qualifiés de journalistes, alors que cette même catégorie ne représente que $15,5 \%$ des accrédités. De la même façon, les professionnels du cinéma constituent 36,3 \% des accrédités, pour 30,4\% des insiders. Enfin, les accrédités non référencés sous une catégorie professionnelle représentent $48 \%$ des festivaliers, alors qu'ils sont 26 \% sur "Cannes Inside ». Même si globalement, cet échantillon semble pouvoir représenter le public cannois, la présence en masse de journalistes est justifiée par le support que constitue Twitter, particulièrement investi par le milieu des médias. Cela donne donc à la population considérée une coloration particulière.

20 Notre analyse portera ici sur le lexique des tweets postés pendant les éditions 2012 et 2013 du Festival de Cannes afin d'envisager comment ce support peut nous amener à éclairer, au moins en partie, les usages festivaliers. Nous avons relevé l'ensemble des termes utilisés dans les tweets des insiders et procédé à une classification par nombre d'utilisations du même terme. L'extraction des données via l'API de Vodkaster nous a fourni une liste de termes associée à un nombre d'occurrences. Nous avons, à partir de cette extraction, réalisé une classification des termes employés selon des catégories qui nous permettent de construire une grille d'analyse des intérêts festivaliers. Cette classification nous a ensuite permis d'organiser le lexique selon des répertoires thématiques.

21 Pour les deux éditions étudiées, on relève une échelle du vocabulaire équivalente avec de 7 à 1881 occurrences d'un même terme pour l'édition 2012 et de 7 à 1672 pour l'édition 2013. Les termes relevés sont largement similaires entre les deux éditions, tant dans les mots que dans le nombre d'occurrences de ceux-ci. Pour chaque édition, l'ensemble des messages postés par les insiders a été pris en compte, sans distinction entre les messages initiaux et les retweets - seul l'élément lexical signifiant le retweet nous en indique d'ailleurs la présence. L'extraction fait donc apparaître un lexique propre au cinéma, au Festival, à des indications géographiques, temporelles, un lexique lié au support, un autre lié aux noms de personnalités du Festival, et une catégorie rassemblant les autres termes. Cette dernière catégorie apparaît comme particulièrement pertinente dans notre analyse car elle met en avant l'importance accordée à certains éléments du quotidien dans la pratique festivalière : le climat - le terme " pluie » pour l'édition 2013 est apparu plus de 20 fois -, les déplacements - le substantif «train » est récurrent sur les deux éditions et apparait entre 20 et 30 fois, au même titre que des mots comme " palmarès ", " cérémonie » ou " compétition », propres au vocabulaire festivalier - ou les lieux de sociabilité - « soirées » apparaît 104 fois dans l'édition 2012, « villa », 54 fois, " Majestic », 24 fois par exemple. La gestion du quotidien tient en effet une place stratégique dans l'expérience festivalière selon les usages que permet le statut de festivalier qui nous est accordé.

22 Enfin, des sous-catégories apparaissent, qui permettent d'affiner cette première lecture quantitative, notamment celui des métiers (acteur, metteur en scène, critique, réalisateur...), qui hiérarchisent et donnent une lecture de l'analyse filmique des 
festivaliers, mais aussi celui des noms des personnalités festivalières, qui donnent une indication sur les cotes de popularité et l'importance accordée à certains statuts : Moretti, président du jury 2012, n'apparaît qu'en 16e position sur 24 avec 21 occurrences ; parallèlement, on retrouvera les premiers noms dans le palmarès du jury : Haneke, Mungiu, Reygadas. Généralement, les stars sont signalées exclusivement avec leur nom de famille ; en revanche, les réalisateurs ou metteurs en scène bénéficient plus souvent de l'association prénom/nom ; enfin, la popularité, ou tout au moins la proximité des personnalités au public, peut être aussi analysée par la présence du prénom seul - une mesure de ce point est permise par un calcul de l'écart entre le nombre d'occurrences pour le nom et celui pour le prénom. Nous avons enfin amorcé une analyse textuelle par la mesure des récurrences verbales pour l'édition 2012 en proposant une classification par nombre d'occurrences globales et une autre par nombre d'occurrences présentes sous des formes différentes. S’il s'avère que « être » et « avoir » tiennent la tête du classement, les autres verbes donnent une image des contenus des messages : faire, dire, savoir, aimer, donner font partie du classement de tête. Il existe enfin tout un lexique des valeurs beaucoup, super, vraiment... - qui est également récurrent au fil des éditions.

La géolocalisation n'est encore que très peu entrée dans les usages et ne permet pas de fournir une image pertinente de la construction d'un territoire et des circulations festivalières. Cependant, la spécificité des tweets est de permettre d'inclure dans chaque message un lien qui provoque un rebond d'information. En effet, la plupart de ces messages permettent un référencement vers d'autres supports en ligne (sites, blogs, autres tweets, photographies, etc.)

Comme nous l'avons déjà évoqué, le visuel de «Cannes Inside » représente un trou de serrure et illustre ainsi le propos porté par ce dispositif, qui est de donner à voir le Festival de Cannes de "l'intérieur ». Cette notion de transgression - que nous avons d'abord analysée comme associée à une possible porosité des cercles de spectateurs du Festival de Cannes - interroge les potentialités disruptives portées par les réseaux sociaux. En effet, Twitter a plusieurs fois été le révélateur de crises sociales, politiques, émergeant d'individualités se positionnant contre un pouvoir en place. On pourrait par construction envisager les usagers de Twitter comme des outsiders (Becker, 1985). Pour autant, si l'on développe cette logique en l'appliquant au Festival de Cannes, est-on encore dans le domaine de la transgression ? Le lexique utilisé par les insiders reste, selon nos observations, plutôt formel et n'exploite que très peu le vocabulaire de la révélation. Certaines polémiques y ont cependant émergé, notamment en 2013, autour de la notion de journalisme et de celle de rumeur rapportée à l'usage de ce réseau.

La codification festivalière très hiérarchisée et formalisée par le système d'attribution des accréditations qui indiquent le niveau d'appartenance de chacun à la société festivalière peut, en effet, être remise en cause par ces réseaux. Le local peut alors communiquer avec le producteur, le cinéphile avec le président du Festival. Généralement, les réseaux favorisent les liens faibles ${ }^{4}$, c'est-à-dire les relations sociales qui ne s'inscrivent ni dans une proximité relationnelle effective ni dans une découverte de l'inconnu, mais dans des relations intermédiaires portées par des communautés d'affinités. En cela, on peut envisager une remise en cause du système cannois par les réseaux sociaux.

Néanmoins, il faut nuancer cette hypothèse par la présence de l'institution festivalière et d'autres communautés de références - Villa des Inrocks, Arte... - dans ces mêmes réseaux. En effet, l'existence d'un compte Twitter officiel du Festival de Cannes donne, dans une certaine mesure, un aval à l'usage de ces réseaux par l'instance qui représente la norme et pondère donc la notion d'outsider numérique.

Pour autant, la façon dont les médias « classiques » exploitent ces ressources d'informations - Le Figaro a notamment construit plusieurs articles autour de l'édition 2013 en se fondant de façon exclusive sur les échanges de tweets - induit que les attentes de polémiques ou de révélations se structurent autour de ces réseaux, et par conséquent, suggère que l'usage de Twitter peut constituer une déviance de l'ordre festivalier établi. 


\section{La posture de leadership et les spectateurs du quatrième cycle}

L'analyse de Twitter par le dispositif « Cannes Inside » nous a permis de relever des récurrences, des limites, et de dessiner un premier périmètre d'une analyse du terrain festivalier par les réseaux sociaux. La finalisation et l'extension de ce modèle d'analyse à la globalité du réseau Twitter doivent pouvoir en faire un véritable terrain d'enquête. En cela, l'API de Twitter permet d'extraire $1 \%$ du flux global de tweets, sur une période donnée. Les données accessibles sont constituées, comme nous l'avons vu avec le dispositif "Cannes Inside », du lexique et du statut, mais elles permettent aussi d'observer les flux d'abonnement et de retweets. Ces informations, absentes de nos premières ressources, permettent une étude de la popularité : popularité du Festival de Cannes sur les réseaux sociaux en cela qu'il est possible, sur les messages fournis, de mesurer l'écart entre le nombre global de messages et ceux spécifiquement en lien avec le Festival de Cannes ; popularité des usagers, par une observation des flux de retweets et de followers avant et après la période festivalière.

Cependant, il nous apparaît nécessaire de poser les limites d'une telle analyse. En effet, Twitter compte 140 millions d'utilisateurs actifs et ce sont 340 millions de messages qui sont postés chaque jour. En France, Twitter représente 5,5 millions d’inscrits, dont 3 millions déclarent être des utilisateurs, avec seulement $13 \%$ d'utilisateurs intensifs (qui postent quotidiennement). Et, comme le note Olivier Donnat, le public des réseaux sociaux est constitué majoritairement d'une population jeune et diplômée5. La porosité et la mobilité sociale induites par ce support doivent donc être pondérées par le fait que ses utilisateurs appartiennent à une catégorie de public elle aussi déterminée socialement.

Plusieurs analyses ont déjà fait état du lien entre la position de leadership et le flux de messages postés. En somme, plus le réseau est investi, plus la popularité augmente, plus les cercles s'élargissent. Il existe donc au sein de ces réseaux une logique de cercles qui va du leader d'opinion construit sur un usage intensif et une pertinence du propos à la posture d'observateur. Il faut aussi pouvoir distinguer l'utilisateur individuel et les comptes officiels - comme celui du Festival de Cannes, qui compte 71905 abonnés et poste à l'année selon un rythme calé sur celui de la préparation de l'événement - qui tirent leur légitimité non pas de la preuve de leur présence sur le réseau, mais de l'image qu'ils véhiculent en amont du réseau.

31 Sur les réseaux sociaux, chaque usager présente et se présente par un statut. Ce statut indique l'identité du membre du réseau selon des critères qui peuvent diverger d'un support à l'autre pour un même individu. Ainsi, Louise « La blogueuse » sur "Cannes Inside », devient Louise Riousse sur Twitter ; elle se définit sur un support comme Stagiaire à la direction des études de la Fémis. Cinéfolle, surtout quand, avec effroi, je pense à tous les films que je n'ai pas vus... Trîptyque Cinéma-Pastaga-Riviera pour la première fois cette année, envoyée par Cinematraque.com - 1e fois à Cannes -, et sur l'autre comme Chroniqueuse pour intermittentedu double-impact @Ciné-folle. Mes tweets n'engagent que toi. La notion de "face » évoqué par E. Goffman peut ici être appliquée au statut proposé par les usagers de Twitter Le statut est le premier pas vers le leadership sur le réseau. Il permet de s'afficher sous le filtre de ce qui pourrait attirer à soi. Pour "Cannes Inside ", il s'agit de préciser sa posture festivalière ; pour Twitter, de marquer son statut social et ses intérêts ; pour les deux, l'humour semble être une valeur sociale positive commune aux usagers des réseaux sociaux.

Comme il est courant de l'observer sur le terrain festivalier, la première expérience festivalière est souvent accompagnée par un guide que l'on suit et qui permet au débutant de construire ses repères cannois ; la position de leadership sur Twitter semble donner cette légitimité de l'expérience même quand elle est toute neuve. 
Extraits des relevés de messages postés sur Twitter via le site Vodkaster :

À partir de demain je m’associe @cannesinside pour vous faire vivre dix jours de

festival enivrants ca va swinguer Le 65e festival de \#cannes commence demain

suivez les péripéties des journalistes d'@artefr avec @bertrand loutte \#cannes2O12

Je vous promets de la folie pendant ce Cannes les twittos jmp/ jgzutw

Cannes c'est aussi ça yfrogcom/ocutrrtbj on me demande des photos d'ambiance ok

Et là ce que l'on ne voit jamais à la télé le palais qui se vide pour fumer avant le film

33 Le dispositif « Cannes Inside " propose donc au public du site de Vodkaster de vivre, en instantané, le Festival de Cannes à travers les profils proposés. Comme en témoignent ces messages relevés sur "Cannes Inside », les insiders jouent le jeu d'une transmission de leur expérience festivalière via ce réseau. Ils invitent même à les suivre promettant des « péripéties », de la « folie », ou de voir « ce que l'on ne voit jamais à la télé ». Cette vie par procuration qu'offre l'usage du réseau Twitter nous semble dessiner un quatrième cercle de spectateurs (Ethis, 2001) qui, hors du territoire du Festival, tentent de vivre l'expérience festivalière non pas par le biais des médias, mais par une identification à un individu qu'ils vont suivre tout au long de leur Festival et avec qui ils vont le partager. Les réseaux constituent ici un moyen, en complément d'autres supports (projection dans les salles de cinéma ou à la télé de la cérémonie de clôture, information par les médias des événements festivaliers), de vivre un quotidien festivalier autre que le sien. Comme les spectateurs du troisième cercle se définissent par leur participation anonyme mais néanmoins active au Festival de Cannes, ceux du quatrième cercle développeraient une façon de vivre le Festival par l'intermédiaire des tweets cannois.

Dans le registre lexical relevé sur «Cannes Inside » pour les éditions 2012 et 2013, le nombre de conversations entre usagers de Twitter peut être retrouvé grâce au signe arobase que mettent les usagers de Twitter dans leurs messages quand ils répondent ou citent quelqu'un. En 2012, nous retrouvons 13 occurrences différentes comportant le signe arobase, en 2013, il s'agit de 28 occurrences. Dans la mesure où nous avons vu que le nombre d'occurrences différentes était stable entre les deux éditions, on peut ici y voir le signe d'une progression qui nous fait dire que les conversations se sont développées pour nos insiders entre 2012 et 2013. En outre, si en 2012, 252 symboles de conversations sont apparus - ce qui classe cette occurrence dans les termes les plus souvent utilisés -, en 2013, nous en comptons 444. Par conséquent, non seulement nous voyons émerger un usage de l'échange de plus en plus courant - nombre de références - mais aussi une fréquence de la discussion plus élevée - nombre total d'occurrences.

Mais au-delà de l'usage de la conversation sur Twitter, nous avons voulu savoir si ces échanges se restreignaient au cercle des insiders ou si le débat s'ouvrait plus largement à un lectorat extérieur. Sur ce point, nous relevons aussi un usage qui s'est développé entre les deux éditions. En effet, en 2012, seules deux discussions mettaient en jeu des usagers non insiders alors qu'en 2013, ce sont six usagers de Twitter extérieurs à "Cannes Inside » qui se sont invités dans les échanges. La progression constatée entre ces deux années indique qu'il existe bien un lectorat des tweets festivaliers. Malgré ce qu'en disent certains insiders.
Extraits des relevés de messages postés sur Twitter via le site Vodkaster :
Comme l'année dernière je prévois une baisse defollowers d'environ $10 \%$ merci de m'avoir suivi dans mes pérégrinations malgré tout
Merci pour les rt puissiez-vous survivre a ces 10 jours de name dropping de hashtags cannes et de villas dont vous vous grattez l'os En route pour \#cannes2013 comme l'année dernière mes tweets "festifs » vont-ils encore me faire perdre la tonne de followers

Les références au lectorat, si elles relèvent d'un autre registre que celles que nous avons mentionnées plus haut, indiquent, elles aussi, la présence des followers - auprès desquels ces insiders s'excusent du manque d'intérêt de leurs tweets festivaliers. Et, même s'ils en évoquent la perte, ils en signalent la présence : «Merci de m’avoir suivi. » Ces messages indiquent à la fois l'existence d'un quatrième cercle de spectateurs et l'importance de ce 
public pour les usagers des réseaux. Ils signalent combien l'existence sur les réseaux sociaux relève de la reconnaissance publique en même temps qu'ils dessinent les premiers contours d'un nouveau cercle de spectateurs cannois. Ils donnent enfin à voir les intérêts et désaveux en évoquant la perte par les tweets " festifs » et « mondains » (name dropping, villas...).

\section{Vers une méthodologie d'analyse des usages festivaliers par les réseaux sociaux}

La fugacité de l'événement festivalier ne permet pas toujours d'en saisir les nuances et demande, dans tous les cas, une observation à long terme. Les traces laissées sur les réseaux sociaux par les publics festivaliers de leur présence cannoise permettent de constituer un corpus exploitable en dehors des temporalités festivalières. Le premier regard que nous avons proposé ici, malgré ses limites, nous permet d'ébaucher la structure d'une grille d'analyse, par le lexique, des ressources fournies par les réseaux sociaux.

L'analyse lexicale nous a amenés à définir une classification des termes employés par les insiders et dessine une organisation des préoccupations et des intérêts festivaliers. La stabilité de la composition des lexiques de chacune des deux éditions étudiées nous amène à envisager que chaque édition peut être lue sous le même filtre et à faire émerger une terminologie du festivalier cannois, mais aussi, à la marge, un champ lexical marqueur des spécificités de chaque édition. Le référencement des champs lexicaux propres à chaque statut d'insider permettrait de pousser cette analyse afin de mettre au jour des postures de festivalier. Enfin, l'étude des interactions via les réseaux sociaux est à envisager dans la construction d'un indicateur de popularité mais aussi dans la définition d'une désorganisation de la société festivalière via les réseaux sociaux.

L'étude des données produites par les insiders nous a permis de soulever trois notions structurantes. Celle d'outsiders numériques, qui demande d'envisager la façon dont la déviance devient constitutive de l'événement - il serait pour celle-ci intéressant de pouvoir analyser la globalité des tweets cannois en dehors de « Cannes Inside » afin de voir si cette déviance construite par « Cannes Inside » se produit « naturellement » sur le réseau -; celle de leadership, pour laquelle nous avons pu constater l'importance du lectorat et de sa fidélité pour les insiders, et qui nous amène à envisager la définition d'un indicateur de popularité croisant les données lexicales produites ici avec les flux d'abonnement et de retweets. Enfin, nous avons évoqué la construction, par les réseaux, d'un quatrième cercle de spectateurs qui construit son expérience festivalière par procuration.

Sur ce dernier, comme nous l'avons indiqué précédemment, seuls $13 \%$ des usagers de Twitter sont actifs. La plupart sont donc membres de ce réseau au titre de simples observateurs et ne sont donc repérables que par le système d'abonnements. La définition de ce public par procuration doit donc s'envisager, au-delà des ressources exploitées ici, par une mesure des flux d'abonnements et de désabonnements des insiders sur la période du Festival de Cannes.

41 Les réseaux sociaux numériques constituent donc une ressource exploitable en termes de données, d'informations, mais ils semblent aussi dessiner un terrain spécifique de l'enquête festivalière. Portant les mêmes caractéristiques d'instantanéité, de discussion et de fort intérêt culturel de leur public que les festivals, les réseaux sociaux possèdent aussi leurs propres publics et leurs propres usages qui croisent ceux des publics festivaliers, les restreignent - être public du festival et usager des réseaux sociaux -, les élargissent - être public des réseaux sociaux mais non festivalier - et offrent une lecture humanisée de l'organisation festivalière observée sur le terrain. Et, si les publics du Festival de Cannes nous ont paru plus sensibles à ces usages numériques (comme nous l'avons expliqué en 
ouverture), d'autres terrains festivaliers, comme les Transmusicales de Rennes (où un tweet wall - dispositif constitué d'un écran installé au cœur de l'espace festivalier et sur lequel sont projetés les tweets liés au hashtag du festival - est devenu le lieu de rassemblement virtuel mais aussi physique de la communauté de festivaliers), peuvent, à notre sens, se prêter à cette trame d'analyse, notamment ceux dont les publics appartiennent aux catégories ayant développé un usage plus généralisé des réseaux sociaux. Car si le mystère du Festival de Cannes - notamment en raison de la réglementation de ses accès - suscite, peut-être plus qu'ailleurs, les curiosités, il n'en reste pas moins vrai que Twitter constitue un outil méthodologique d'analyse des événements culturels pour les sociologues et un moyen d'accès à la connaissance et à l'expérience festivalières pour les publics.

\section{Bibliographie}

Becker (Howard). 1985. Outsiders. Études de sociologie de la déviance. Paris : Éditions Métailié, coll. « Leçons de choses ».

Cardon (Dominique). 2013. "Liens faibles et liens forts sur les réseaux sociaux ». Les Cahiers français, 372, p. 61-65. Paris : La Documentation française.

Collins (Randall C.). 2000. The Sociology of Philosophies: A Global Theory of Intellectual Change. Cambridge : Belknap Press of Harvard University Press.

Donnat (Olivier). 2009. " Les pratiques culturelles des Français à l'ère numérique : Éléments de synthèse 1997-2008 ». Culture études. Paris : Département des études de la prospective et des statistiques (DEPS).

Ethis (Emmanuel) (dir.). 2001. Aux marches du palais. Le Festival de Cannes sous le regard des sciences sociales. Paris : Département des études de la prospective et des statistiques (DEPS), coll. "Questions de culture».

Goffman (Erving). 1974. Les Rites d’interaction. Paris : Les Éditions de Minuit, coll. « Le sens commun ».

Katz (Elihu) \& Lazarsfeld (Paul). 1955. Personal Influence: The Part Played by People in the Flow of Mass Communications. Glencoe : The Free Press.

Scherer (Éric). 2009. La Révolution numérique : Glossaire. Paris : Dalloz-Sirey.

\section{Notes}

1 Ethis (Emmanuel) \& Poli (Marie-Sylvie). « Hopper 2013/Cannes 2013 : Au prisme des écritures numériques », 24, p. 63 in Culture \& Musées sous la direction de Damien Malinas. Arles : Actes Sud.

2 «Les réseaux sociaux signent moins la fin de la vie privée, qu’ils ne sont le théâtre de nouvelles formes expressives dans lesquelles le personnel fait l'objet d'une mise en scène soigneusement calculée. » (Cardon, 2013).

3 Applications Programming Interface : une interface de programmation est un ensemble de fonctions, procédures, protocoles, structures de données, mis à disposition des programmes informatiques pour soutenir la construction d'un service plus riche. L'ouverture des API donne la possibilité à des tiers d’utiliser les infrastructures déjà développées. (Scherer, 2009).

4 « Ce que les réseaux sociaux numériques transforment le plus dans l'organisation sociale des individus n'est ni le contact aventureux avec des inconnus, ni l'échange intime avec les plus proches. C'est entre les deux, au sein du répertoire des "liens faibles", ces indispensables intermédiaires de la vie sociale que se déploie la nouvelle sociabilité numérique. » (Cardon, 2013).

5 « Les jeunes et les milieux favorisés sont les principaux utilisateurs des nouveaux écrans, à la différence de la télévision dont la consommation a toujours été le fait des personnes âgées et peu diplômées. La profonde originalité de l'Internet tient dans ce paradoxe : bien qu'utilisé très largement à domicile - les connexions sur appareils nomades restant à ce jour limitées -, ce nouveau média apparaît plutôt lié à la culture de sortie dont sont porteuses les fractions jeunes et diplômées de la population, celles dont le mode de loisir est le plus tourné vers l'extérieur du domicile et dont la participation à la vie culturelle est la plus forte. » (Donnat, 2009). 


\title{
Pour citer cet article
}

Référence papier

Myriam Dougados, Jean-Louis Fabiani, Julien Gaillard et Yonathan Portilla, « Les usages de

Twitter au festival de Cannes. Pratiques numériques et construction des opinions

esthétiques », Culture \& Musées, 24 | 2014, 89-106.

Référence électronique

Myriam Dougados, Jean-Louis Fabiani, Julien Gaillard et Yonathan Portilla, « Les usages de

Twitter au festival de Cannes. Pratiques numériques et construction des opinions

esthétiques », Culture \& Musées [En ligne], 24 | 2014, mis en ligne le 19 juin 2018, consulté le 20

juin 2021. URL : http://journals.openedition.org/culturemusees/639 ; DOI :

https://doi.org/10.4000/culturemusees.639

\section{Auteurs}

\section{Myriam Dougados}

Myriam Dougados est doctorante en Sciences de l'information et de la communication à l'université d'Avignon et des Pays de Vaucluse au sein de l'équipe Culture et Communication du centre Norbert Élias UMR 8562, sous la direction d'Emmanuel Ethis. Ses travaux portent sur les traces de l'enfance au Festival de Cannes. Dougados (Myriam). « Jeu de cache-cache. Les phénomènes de l'enfance au Festival de Cannes », in Problématisation et Méthodologie de recherche, Actes des Journées doctorales 2011, Bordeaux, 30-31 mars 2011, Société française des Sciences de l'information et de la communication, mars 2012, p. 105.

myriam.dougados@univ-avignon.fr

\section{Jean-Louis Fabiani}

Jean-Louis Fabiani, professeur à la Central European University de Budapest, directeur d'étude de l'École des hautes études en sciences sociales (EHESS), est rattaché au centre d'études sociologiques et politiques Raymond Aron (CNRS / EHESS).

Ses recherches portent notamment sur l'étude des « configurations de savoir », entendues comme les manières dont les disciplines et les institutions savantes se construisent et se modifient. Parallèlement, il a été l'un des premiers à contribuer à la sociologie de l'environnement en France et il se consacre également à des recherches en sociologie de la culture. Il a été plusieurs fois professeur invité aux États-Unis, notamment par les départements de sociologie de l'université de Chicago et de l'université du Michigan. Il est également professeur associé à l'université de Montréal (Québec). Fabiani (Jean-Louis). 2010. Qu'est-ce qu'un philosophe français ? La vie sociale des concepts. Paris : Éditions de l'EHESS. fabianij@ceu.hu

\begin{abstract}
Julien Gaillard Julien Gaillard est doctorant en informatique (spécialité Machine Learning, Big Data, Automatic Opinion Analysis) à l'université d'Avignon et des Pays de Vaucluse (SFR Agorantic), au sein du laboratoire d'informatique d'Avignon (LIA), sous la direction de Marc El-Bèze, Emmanuel Ethis et Eitan Altman. Ses travaux portent sur la conception d'un système de recommandations intégrant deux fonctionnalités innovantes : un nouvel algorithme real-time d'une part (mise à jour instantanée, « en temps réel »), capable de produire des recommandations personnalisées et argumentées textuellement d'autre part. Julien Gaillard a présenté ses travaux lors de conférences internationales à Orlando (INFOCOM 2012), Seattle (EMNLP 2013) et Las Vegas (DMIN 2013).

julien.gaillard@univ-avignon.fr
\end{abstract}

\section{Yonathan Portilla}

\section{Droits d'auteur}

Culture \& Musées 